\title{
BMJ Open Variation in cardiovascular disease care: an Australian cohort study on sex differences in receipt of coronary procedures
}

\author{
Alexandra Jane Fogg, ${ }^{1}$ Jennifer Welsh, ${ }^{\circledR 2}$ Emily Banks, ${ }^{2,3}$ Walter Abhayaratna, ${ }^{1,4}$ \\ Rosemary $\mathrm{J} \mathrm{Korda}^{2}$
}

To cite: Fogg AJ, Welsh J, Banks $\mathrm{E}$, et al. Variation in cardiovascular disease care: an Australian cohort study on sex differences in receipt of coronary procedures. BMJ Open 2019;9:e026507. doi:10.1136/ bmjopen-2018-026507

- Prepublication history and additional material for this paper are available online. To view these files, please visit the journal online (http://dx.doi. org/10.1136/bmjopen-2018026507).

Received 5 September 2018 Revised 15 March 2019 Accepted 17 June 2019

Check for updates

(c) Author(s) (or their employer(s)) 2019. Re-use permitted under CC BY-NC. No commercial re-use. See rights and permissions. Published by BMJ.

${ }^{1}$ Australian National University Medical School, The Australian National University, Canberra, Australian Capital Territory, Australia

${ }^{2}$ National Centre for Epidemiology and Population Health, Research School of Population Health, The Australian National University, Canberra, Australian Capital Territory, Australia

${ }^{3}$ The Sax Institute, Sydney, New South Wales, Australia

${ }^{4}$ Division of Medicine, Canberra Hospital, Canberra, Australian Capital Territory, Australia

Correspondence to

Dr Rosemary J Korda;

rosemary.korda@anu.edu.au

\section{ABSTRACT}

Objectives The aim of this study was to quantify sex differences in diagnostic and revascularisation coronary procedures within 1 year of hospitalisation for acute myocardial infarction (AMI) or angina.

Design This is a prospective cohort study. Baseline questionnaire (January 2006-April 2009) data from the Sax Institute's 45 and Up Study were linked to hospitalisation and mortality data (to 30 June 2016) in a time-to-event analysis, treating death as a censoring event.

Setting This was conducted in New South Wales, Australia.

Participants The study included participants aged $\geq 45$ years with no history of ischaemic heart disease (IHD) who were admitted to hospital with a primary diagnosis of AMI $(n=4580)$ or a primary diagnosis of angina or chronic IHD with secondary diagnosis of angina $(n=4457)$.

Outcome measures The outcome of this study was coronary angiography and coronary revascularisation with percutaneous coronary intervention or coronary artery bypass graft (PCl/CABG) within 1 year of index admission. Cox regression models compared coronary procedure rates in men and women, adjusting sequentially for age, sociodemographic variables and health characteristics. Results Among patients with AMI, $71.6 \%$ of men (crude rate $3.45 /$ person-year) and $64.7 \%$ of women (2.62/personyear) received angiography; $57.8 \%$ of men (1.73/personyear) and $37.4 \%$ of women (0.77/person-year) received PCI/CABG. Adjusted HRs for men versus women were 1.00 (0.92-1.08) for angiography and 1.51 (1.38-1.67) for PCI/ CABG. In the angina group, $67.3 \%$ of men (crude rate 2.36 / person-year) and $54.9 \%$ of women (1.32/person-year) received angiography; $44.6 \%$ of men $(0.90 /$ person-year) and $19.5 \%$ of women $(0.26 /$ person-year) received $\mathrm{PCl} /$ CABG. Adjusted HRs were 1.24 (1.14-1.34) and 2.44 (2.16-2.75), respectively.

Conclusions Men are more likely than women to receive coronary procedures, particularly revascularisation. This difference is most evident among people with angina, where clinical guidelines are less prescriptive than for AMI.

\section{BACKGROUND}

Cardiovascular disease (CVD) is the leading cause of morbidity and mortality worldwide, ${ }^{1}$
Strengths and limitations of this study

- This study uses population-based survey data linked to routinely collected health data.

- This study has a relatively large number of participants, with virtually complete capture of procedures.

- This study has adjusted for a large range of baseline sociodemographic and health factors; however, clinical factors on presentation were not included.

- While diagnosis of acute myocardial infarction is highly valid within hospital data, there is relatively low concordance for angina diagnoses, with possible over-representation or under-representation of rates.

- The study cohort, while broadly representative of the general population, is likely to be healthier and have lower hospitalisation and mortality rates than their peers.

and a leading cause of death in Australia. ${ }^{2}$ While incidence of CVD is higher among men, ${ }^{3}$ in many parts of the world, women experience worse outcomes. ${ }^{4}$ Coronary interventions, including percutaneous coronary intervention (PCI) and coronary artery bypass grafting (CABG), contribute to improved outcomes after an acute coronary event in both men and women. ${ }^{4}$ Despite the significant disease burden and availability of effective interventions, CVD in women as a whole remains underdiagnosed and less aggressively treated. ${ }^{5}{ }^{6}$ Women are under-represented in clinical trials, ${ }^{6}$ and there are important gaps in the evidence for recognition and treatment of adverse coronary events.

It is known that incident acute myocardial infarction (AMI) and angina pectoris present, on average, 7-10 years later in women compared with men. ${ }^{6} 7$ Women generally present to hospital older and with a greater number of risk factors than men, ${ }^{6}$ experiencing higher mortality and reinfarction rates after a first AMI. ${ }^{7}$ Disparities in care 
received by women compared with men have been documented in the USA, even when accounting for income, education and site of care. ${ }^{8}$ Evidence from Europe and the USA demonstrate that women with CVD are less likely to undergo diagnostic angiography or intervention with PCI or CABG, ${ }^{67}$ procedures with documented clinical benefit. $^{45}$

There is limited information on sex differences in cardiovascular care in Australia, including diagnosis and management with coronary procedures. Age-standardised rates of procedures are lower among women compared with men; ${ }^{9}$ however, underlying CVD and other factors related to delivery of care are not taken into consideration in these figures. Hence, the extent to which these differences in rates reflect inequalities in care is uncertain. This study aimed to quantify sex differences in care delivery by comparing coronary procedure rates in men and women admitted with AMI or angina, adjusting for other sociodemographic and health-related factors.

\section{METHODS}

\section{Data sources}

Data were obtained from the Sax Institute's 45 and Up Study, a population-based cohort study involving 267153 men and women aged 45 and older from New South Wales (NSW) Australia. The 45 and Up Study participants were randomly selected from the Department of Human Services (formerly Medicare Australia) database, Australia's universal health insurance system, with oversampling of individuals living in rural areas and those over the age of 80 by a factor of 2 . All individuals living in remote areas were invited to participate. Participants enrolled by completing a mailed self-administered questionnaire and provided signed consent for long-term follow-up and data linkage with a range of health databases. Approximately $10 \%$ of the NSW population aged 45 and older were included in the sample, an overall response rate of $18 \%$. The Study is described in detail elsewhere, ${ }^{10}$ with questionnaires available online. ${ }^{11}$

Baseline data from the 45 and Up Study participants were linked to hospital data from the NSW Admitted Patient Data Collection (APDC, from 1 July 2000 to 30 June 2016) and death data from the NSW Registry of Births, Deaths and Marriages and the National Death Index (1 January 2006 to 30 June 2016). The latter was linked by the Australian Institute of Health and Welfare. Included in the APDC is a record of all hospitalisations in NSW, dates of admission and discharge, and reason for admission. International Statistical Classification of Diseases and Related Health Problems, Tenth Revision, Australian Modification (ICD-10-AM) and Australian Classification of Health Interventions codes are incorporated into the APDC, with each record containing up to 51 diagnosis and 50 procedure codes. Death data included date of death (used for censoring as per time-toevent analysis) based on death from any cause.
Data were linked probabilistically by the Centre for Health Record linkage using personal information (full name, date of birth, sex and address). It is likely that during the follow-up period a small but unknown number of participants moved interstate. Although hospitalisations in neighbouring states are not included, these are estimated to make up fewer than $2 \%$ of admissions in NSW residents. Follow-up for hospitalisations is considered to be $\sim 98 \%$ complete among those who continue to live in NSW. Quality assurance data on the data linkage show false-positive and false-negative rates of $<0.5 \%$ and $<0.1 \%$, respectively.

\section{Study population}

All 45 and Up Study participants admitted to hospital with a primary diagnosis of AMI or angina (stable or unstable) after entry into the 45 and Up Study were included in the sample. Those with a primary diagnosis of chronic ischaemic heart disease (IHD) and secondary diagnosis of angina were also included due to the possibility of patients with angina being admitted for elective revascularisation under these diagnostic codes. ICD-10-AM diagnosis codes I21 (AMI), I20 (angina pectoris) and I25 (chronic IHD) were used to ascertain admission. Note that these are clinicopathological diagnoses, with angina specifically referring to chest pain from insufficient myocardial oxygenation and coronary artery disease. I21 coding for AMI includes both ST-elevation and non-ST elevation myocardial infarction, with current ICD-10 coding unable to reliably distinguish between these. ${ }^{12}$

Participants with a prior history of IHD were excluded, defined as self-reported heart disease on the baseline questionnaire and/or admission to hospital for IHD (120-125), and/or a related interventional procedure (angiogram, PCI or CABG-defined below), as ascertained by diagnosis and procedure code fields of APDC in the 6 years prior to entering the 45 and Up Study.

\section{Variables}

The study outcomes were investigation with angiography, and coronary intervention with PCI or CABG, within 12 months of index admission to hospital.

Outcomes were ascertained using all 50 APDC procedure-code fields, coded using the Australian Classification of Health Interventions which is used in conjunction with ICD-10-AM ${ }^{13}$ : angiography $(38215,38218)$, PCI (35 304 to 00, 35 305-00, 35 304-01, 35 305-01, 38300-00, 38303-00 (block: 670), 35 310-00, 35 310-01, 35310-02, 35310-03, 35310-05, 38306-00, 38306-01, 38306-02, 38306-03, 38306-05 (block: 671)) and CABG (38497-00 to $38497-07,38500-00$ to $38500-04,38503-00$ to 38503-04, 90201-00 to 90201-03, 38500-05, 38503-05 (blocks 672-679)). Italicised codes have been included to reflect changes in procedure coding during the follow-up period.

The main exposure of interest was sex (male or female), self-reported on the baseline questionnaire. Sociodemographic and health characteristics that may confound/ 
mediate the relationship between sex and receipt of coronary procedures were also measured on the baseline questionnaire. These included country of birth (Australia/New Zealand or other), region of residence (major city, inner regional or outer regional/remote/ very remote), highest qualification (no school certificate, school/trade certificate or diploma, or tertiary degree), private health insurance (no private health insurance, or private hospital insurance/Department of Veterans Affairs health card), marital status (married/de facto or not), obesity (body mass index (BMI) $\geq 30 \mathrm{~kg} / \mathrm{m}^{2}$, or not, based on self-reported height and weight), physical functioning (no/minor limitations, moderate limitations or severe limitations, based on levels of functional limitation, as adapted from the Medical Outcomes Score Physical Functioning Subscale ${ }^{14}$ ) and psychological distress (low $(10-<16)$, moderate $(16-<22)$ or high $(22-50)$, as per the Kessler 10). ${ }^{15}$

\section{Analysis}

Cox proportional hazard regression was used to model the association between sex and receipt of coronary procedures. For each analysis, participants contributed person-years from the date of index admission for AMI or angina until either the specified outcome of interest, death from any cause or end of follow-up (30 June 2016), whichever was the earliest, to a maximum of one calendar year. Data from patients in the angina sample were also censored if they were subsequently admitted with AMI. Proportional hazards assumption was tested, with the $\mathrm{p}$-value set a priori to $\mathrm{p}<0.01$. All analyses were conducted separately for patients whose index admission was for AMI, and for those whose index admission was for angina. Patients presenting concurrently with AMI and angina were included in the AMI sample.

For each outcome, we calculated crude incidence rates separately for men and women, then ran a series of Cox regression models to estimate HRs in relation to sex. Model 1 was adjusted for age (5-year age categories from 45 to 54 years through to $\geq 80$ years). Model 2 was adjusted for age and sociodemographic variables (country of birth, region of residence, highest qualification, private health insurance and marital status). Model 3 was further adjusted for additional baseline health characteristics (obesity, physical functioning and psychological distress). Participants with missing values for covariates were included in the models, with missing coded as a separate category.

Multiple sensitivity analyses were performed. First, we used a maximum follow-up period of 30 days after index admission rather than 12 months. Second, we used an alternative indicator of baseline health-self-rated health, measured with three categories (excellent/very good, good, fair/poor)-instead of functional limitation, obesity and psychological distress (model 3). Third, we additionally controlled for comorbidity using the Charlson Comorbidity Index, ${ }^{16}$ using all diagnostic codes in the 12 months prior to the index admission, with scores categorised as 0,1 or $\geq 2$. Fourth, we restricted analysis of the patients with angina to those admitted with a primary diagnosis of unstable angina (ICD-10: I20.0). Further sensitivity analyses were performed, excluding patients who were aged 85 and older, and then controlling for additional patient factors of smoking, self-rated diabetes, treatment for high blood pressure, treatment for high cholesterol and family history of heart disease.

Analyses were performed using Stata V.14. ${ }^{17}$

\section{Patient and public involvement}

The 45 and Up Study has involved community and consumer representation from its inception, through its early-phase Community and Ethical Oversight Committee, and through engagement with its participants, who constitute $10 \%$ of the NSW general population in the target age range. Participants are regularly informed of Study projects through newsletters and the Study website. The specific analyses in this project are part of a general initiative from the Heart Foundation of Australia on women and heart disease and have received consumer input through this organisation. Preliminary results were presented at a Heart Foundation event involving the general public, and further dissemination of results to the public is expected.

\section{RESULTS}

A total of 9037 patients were included in the study: 4580 admitted with AMI and 4457 admitted with angina. There were no patients in the sample who had IHD as a primary diagnosis and angina as a secondary diagnosis. Sample characteristics are shown in table 1 . The profiles of patients within the AMI and angina groups were similar, with a few exceptions. Notably, those in the angina group were less likely to be aged 85 and older $(8.56 \%$ vs $19.4 \%)$ and more likely to have private health insurance at baseline $(64.6 \%$ vs $56.9 \%)$ than those in the AMI group. There was a greater percentage of men within both AMI and angina groups, $63.6 \%$ and $56.2 \%$, respectively.

\section{Coronary procedures in patients with AMI}

Among those admitted to hospital with AMI, 69.1\% received angiography, $71.6 \%$ of men vs $64.7 \%$ of women, and $50.4 \%$ underwent PCI/CABG, $57.8 \%$ of men vs $37.4 \%$ of women, respectively. The proportion of patients with AMI who survived the 1-year follow-up period was $86.9 \%$ (88.6\% men vs $84.0 \%$ women). Among these people, $75.6 \%$ received angiography $(77.6 \%$ vs $71.9 \%)$ and $55.4 \%$ underwent PCI/CABG (63.2\% vs $41.0 \%)$.

Crude rates per person-year of angiography were 3.45 (3.31-3.60) and 2.62 (2.47-2.78) in men and women, respectively; and crude rates of $\mathrm{PCI} / \mathrm{CABG}$ were 1.73 (1.64-1.81) and 0.77 (0.71-0.83), respectively (table 2).

Cox models showed angiography rates were similar in men and women, with no difference after adjustment for sociodemographic and health variables $(\mathrm{HR}=1.00$, 0.92-1.08) (table 2). In contrast, rates for PCI/CABG 
Table 1 Characteristics of patients admitted to hospital with acute myocardial infarction (AMI) or angina by sex

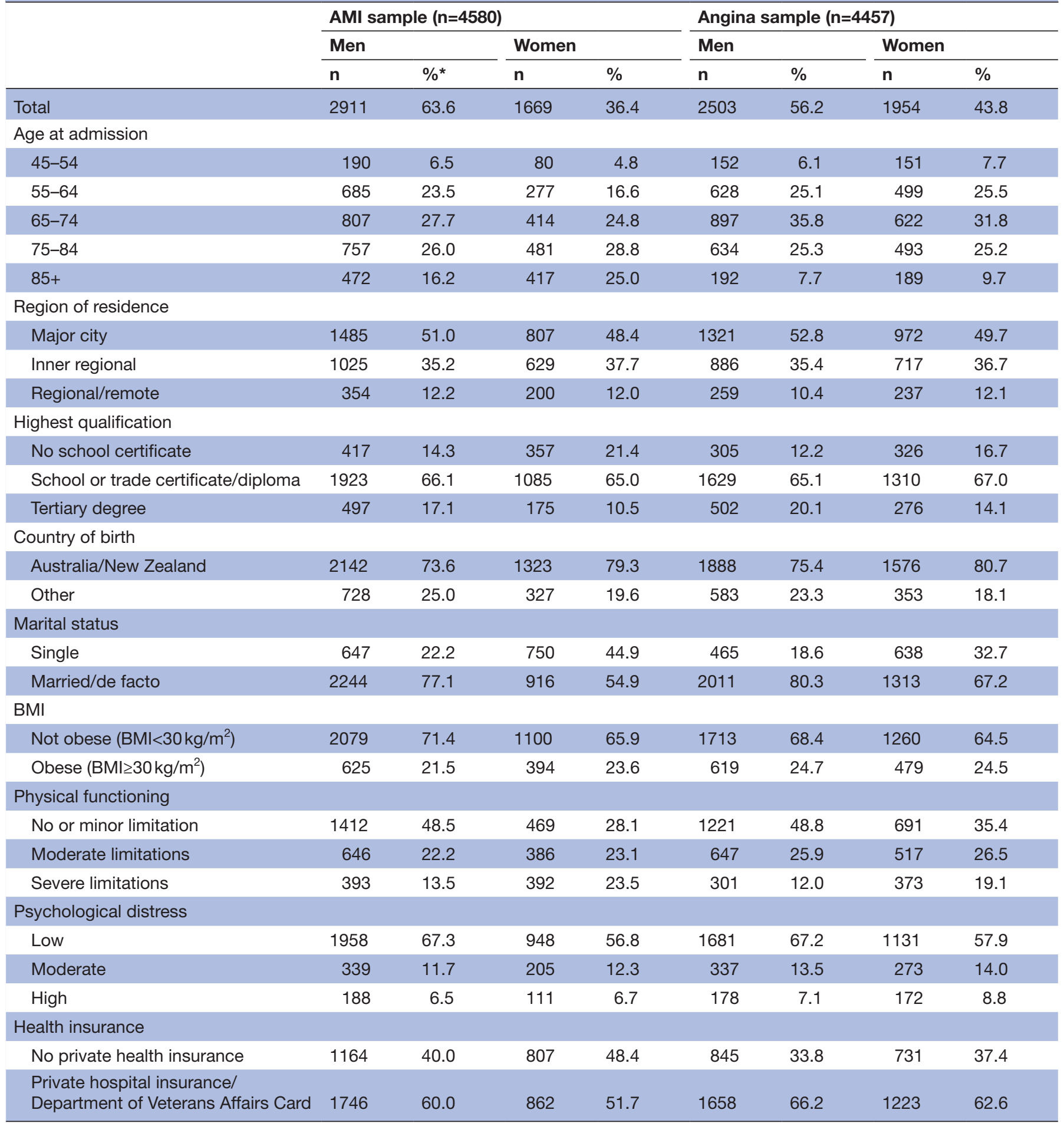

*Pecentage of missing cases: AMI sample (men, women): region of residence (1.6, 2.0); highest qualification (2.5, 3.1); country of birth $(1.4,1.1)$; marital status $(0.7,0.2)$; body mass index (BMI) $(7.1,10.5)$; physical limitations $(15.8,25.3)$; psychological distress (14.6, $24.3)$; health insurance $(<0.1,0.0)$. Angina sample (men, women): region of residence $(1.5,1.4)$; highest qualification $(2.7,2.2)$; country of birth $(1.3,1.3)$; marital status $(1.1,0.1)$; $\operatorname{BMI}(6.8,11.0)$; physical limitations $(13.3,19.1)$; psychological distress $(12.3$, 19.3); health insurance $(0.0,0.0)$.

were around $50 \%$ higher in men than women (adjusted $\mathrm{HR}=1.51,1.38-1.67)$.

\section{Coronary procedures in patients with angina}

Among those admitted to hospital with angina, $61.9 \%$ received angiography, $67.3 \%$ of men vs $54.9 \%$ of women, and $33.6 \%$ underwent PCI/CABG, $44.6 \%$ of men vs $19.5 \%$ of women, respectively. The proportion of patients with angina who survived to the end of the 1-year follow-up period was $97.4 \%$ (96.9\% men vs $98.0 \%$ women). Among these people, $62.4 \%$ received angiography $(68.1 \%$ vs 
Table 2 Rates of coronary procedures within 1 year of admission with AMI by sex and associated HRs ( $\mathrm{n}=4580)$

\begin{tabular}{|c|c|c|c|c|c|}
\hline & Procedures/py & $\begin{array}{l}\text { Crude rate per py } \\
(95 \% \mathrm{Cl})\end{array}$ & $\begin{array}{l}\text { Model } 1 \\
\text { HR (95\% Cl) }\end{array}$ & $\begin{array}{l}\text { Model } 2 \\
\text { HR (95\% Cl) }\end{array}$ & $\begin{array}{l}\text { Model } 3 \\
\text { HR (95\% Cl) }\end{array}$ \\
\hline \multicolumn{6}{|c|}{ Angiography } \\
\hline Women & $1079 / 411.7$ & 2.62 (2.47 to 2.78 ) & 1.00 & 1.00 & 1.00 \\
\hline \multicolumn{6}{|l|}{ PCI/CABG } \\
\hline
\end{tabular}

Notes: Model 1 is adjusted for age at first admission. Model 2 is adjusted for age and sociodemographic variables (country of birth, region of residence, highest qualification, health insurance, marital status). Model 3 is adjusted for age, sociodemographic variables and health-related variables (obesity, physical functioning, psychological distress).

CABG, coronary artery bypass graft; $\mathrm{PCl}$, percutaneous coronary intervention; py, person-year.

$55.1 \%)$ and $33.9 \%$ underwent PCI/CABG (45.3\% vs $19.4 \%)$.

Crude rates per person-year for angiography were 2.36 (2.25-2.47) and 1.32 (1.24-1.40) for men and women, respectively; and crude rates for PCI/CABG were 0.90 (0.85-0.95) and $0.26(0.24-0.29)$, respectively (table 3 ).

Cox models showed angiography rates were around $25 \%$ higher among men after adjusting for all factors $(\mathrm{HR}=1.24,1.14-1.34)$. Rates for PCI/CABG in men were around $150 \%$ higher than those of women (adjusted $\mathrm{HR}=2.44,2.16-2.75$ ).

There were no violations of the proportional hazards assumption for the sex variable in the models with 12 months of follow-up.

Sensitivity analyses using a 30-day follow-up period produced almost identical HRs (online supplementary tables S1-S2). While violations of the proportional hazards assumption were found on testing in both the AMI and the angina samples, there were no major violations detected in log-log plots. HRs were not materially different from those in the main analysis when adjusted for self-rated health as an alternative indicator of health (online supplementary tables S3-S4), when additionally adjusted for the Charlson Comorbidity Index (online supplementary tables S5-S6) or when estimated on a sample restricted to patients admitted with a primary diagnosis of unstable angina (online supplementary table S7). Similarly, HRs were not materially different from those in the main analysis when participants 85 and older were excluded from the sample (online supplementary tables S8 (patients with AMI) and S9 (patients with angina)) and when additionally adjusting for smoking, self-rated diabetes, treatment for high blood pressure, treatment for high cholesterol and family history of heart disease (online supplementary table S10).

\section{DISCUSSION}

Our findings demonstrate clear sex differences in receipt of coronary procedures. Men were more likely to receive coronary revascularisation (PCI/CABG) for the management of AMI or angina. Differences in revascularisation rates were most pronounced among those admitted with angina, among whom men were also more likely to undergo diagnostic angiography.

This study uses Australian data, with findings generally consistent with published evidence internationally. There is evidence from the USA and Sweden that men are more likely than women to receive revascularisation procedures such as PCI or CABG after hospital

Table 3 Rates of coronary procedures within 1 year of admission with angina by sex and associated HRs $(n=4457)$

\begin{tabular}{|c|c|c|c|c|c|}
\hline & Procedures/py & $\begin{array}{l}\text { Crude rate per py } \\
(95 \% \mathrm{Cl})\end{array}$ & $\begin{array}{l}\text { Model } 1 \\
\text { HR (95\% Cl) }\end{array}$ & $\begin{array}{l}\text { Model } 2 \\
\text { HR (95\% Cl) }\end{array}$ & $\begin{array}{l}\text { Model } 3 \\
\text { HR }(95 \% \mathrm{Cl})\end{array}$ \\
\hline \multicolumn{6}{|c|}{ Angiography } \\
\hline Women & $1072 / 814.3$ & 1.32 (1.24 to 1.40$)$ & 1.00 & 1.00 & 1.00 \\
\hline \multicolumn{6}{|l|}{$\mathrm{PCl} / \mathrm{CABG}$} \\
\hline Women & $381 / 1450.8$ & 0.26 (0.24 to 0.29$)$ & 1.00 & 1.00 & 1.00 \\
\hline
\end{tabular}

Notes: Model 1 is adjusted for age at first admission. Model 2 is adjusted for age and sociodemographic variables (country of birth, region of residence, highest qualification, health insurance, marital status). Model 3 is adjusted for age, sociodemographic variables and health-related variables (obesity, physical functioning, psychological distress).

CABG, coronary artery bypass graft; $\mathrm{PCl}$, percutaneous coronary intervention; py, person-year. 
admission with AMI or angina. ${ }^{718}$ Our finding that rates of PCI/CABG among men were 1.5 and 2.4 times those for women for AMI and angina, respectively, is consistent with the above studies. A cohort study from the UK demonstrated this relationship for CABG, with men having twice the odds of receiving CABG than women $(\mathrm{OR}=1.90,1.21-3.00)$; however, no difference in overall revascularisation rates was found. ${ }^{19}$ Evidence regarding angiography is less consistent. There is evidence from the USA, including a review, that suggests sex differences exist ${ }^{2021}$; however, these considered acute coronary syndrome as a whole rather than AMI and angina separately. No significant difference was found in the UK for receipt of CVD investigations, including angiography. ${ }^{19}$ This is consistent with our findings on angiography in patients with AMI, but differ from our findings in patients with angina where we found a $25 \%$ difference in rates between men and women.

Factors driving the observed sex differences in procedure rates are not known; however, likely contributors include differences in clinical presentation. There are established sex differences in the pathophysiology, diagnosis and outcome of therapies related to AMI and angina. ${ }^{7}$ For example, greater proportions of small-vessel coronary disease among women, including Takotsubo cardiomyopathy and other forms of myocardial infarction with non-obstructive coronary arteries, ${ }^{22}$ could contribute to lower rates of PCI/CABG after AMI. Previous studies have shown that women who present with AMI or stable angina are more likely to have non-obstructive coronary artery disease compared with men, that is, $<50 \%$ stenosis of coronary arteries. ${ }^{23} 24$

After CABG, women experience higher complication rates and increased mortality compared with men, a finding that is more pronounced in younger age groups. ${ }^{6}$ While this may contribute to the lower rate of procedures among women, the directionality of this relationship cannot be assumed.

The use of questionnaire data linked to large-scale routine data enabled a wide range of personal characteristics and other factors to be included in models, such as socioeconomic position and physical functioning; however, clinical factors relating to patient presentation and symptom severity were not available in the dataset. A US review, however, concluded that clinical factors do not fully explain the discrepancies in procedure rates between sexes. ${ }^{25}$ Thus, while we cannot exclude the possibility of the sex differences representing appropriate clinical care, this seems an unlikely explanation for the total observed variation in procedure rates, with multiple factors likely at play. ${ }^{25}$

Another possible explanation for our findings is unwarranted variation due to discrimination. This includes the possibility of unconscious gender bias, with one systematic review demonstrating implicit bias towards patients by healthcare professionals. ${ }^{26}$ Australia's universal health system, providing free access to hospital care, should present few barriers to receiving equitable care. There are clear guidelines for the use of coronary procedures among patients presenting with AMI; however, the use of procedures for those presenting with angina is not as clear. ${ }^{27}$ Sex differences found in receipt of coronary procedures, particularly in cases of angina, reinforce the suggestion that these differences reflect an inequality in care. This raises two distinct issues. First, the possible underuse of coronary procedures in women, which may indicate that the healthcare needs of a portion of Australians are not being adequately met, and second, the possible overuse of coronary procedures in men, which raises the question of waste within a healthcare system with limited resources. ${ }^{27}$

Strengths of this study include the use of population-based survey data linked to routinely collected data. The number of participants included was relatively large, with virtually complete capture of procedures. Questionnaire and other data permitting, there was adjustment for a large range of baseline sociodemographic and health factors (most of which are not included in administrative data). Additionally, the diagnosis of AMI is highly valid within hospital data, with sensitivity and specificity of $\geq 86 \%$ and a positive predictive value of $\geq 93 \%,{ }^{28}$ and high concordance between diagnostic codes and physician review. ${ }^{29}$ This is not true for angina, however, where concordance is relatively low. ${ }^{29}$ This may have led to an overestimation or underestimation of procedure rates for angina. As only those admitted to hospital were included in this study, it is possible that some participants with angina, who could benefit from a coronary procedure, were not captured. While the 45 and Up Study cohort is broadly representative of the general population, participants are likely to be healthier and have lower hospitalisation and mortality rates than others in this age group, consistent with the healthy cohort effect. ${ }^{30}$ However, while this may mean that the absolute rates of coronary procedures among those with coronary heart disease in this study differ from those of the general population, internal comparisons are unlikely to be influenced by this bias; hence, relative rates in relation to sex are likely to remain valid. ${ }^{31} 32$

\section{CONCLUSIONS}

CVD is a national health priority for Australia, with substantial morbidity and mortality. This study showed that men are more likely than women to receive coronary revascularisation procedures after admission to hospital with an AMI or angina. This relationship was particularly evident among the angina group, for whom clinical guidelines are less clear. While we cannot exclude that this discrepancy reflects appropriate care due to differences in clinical presentation, we must consider the possibility that sex differences, accounting for sociodemographic position and health-related factors, may indicate that the healthcare needs of a portion of the Australian population are not being adequately met. Morbidity and mortality among Australian women may be unnecessarily 
increased due to not receiving coronary intervention after AMI or angina. Alternatively, relative overuse in men cannot be ruled out. Either way, there is potential for health gain in elucidating and addressing this sex difference in receipt of coronary intervention, increasing awareness and delivery of best practice care.

Acknowledgements This research was completed using data collected through the 45 and Up Study (www.saxinstitute.org.au). The 45 and Up Study is managed by the Sax Institute in collaboration with major partner Cancer Council NSW; and partners: the National Heart Foundation of Australia (NSW Division); NSW Ministry of Health; NSW Government Family \& Community Services - Ageing, Carers and the Disability Council NSW; and the Australian Red Cross Blood Service. We thank the many thousands of people participating in the 45 and Up Study. We also acknowledge the assistance of the Centre for Health Record Linkage.

Contributors AJF provided input into the study design, performed statistical analysis, interpreted the data and drafted the manuscript. JW provided input into the study design, performed statistical analysis and reviewed the manuscript. EB provided input into the study design and reviewed the manuscript. WA reviewed the manuscript. RK conceived and designed the study and assisted in writing the manuscript. All authors read and approved the final manuscript.

Funding This research was supported by a NSW CVRN Women and Heart Disease grant from the National Heart Foundation of Australia (101692). EB is supported by the National Health and Medical Research Council of Australia (1042717).

Competing interests None declared.

Patient consent for publication Not required.

Ethics approval Ethics approvals for this project were obtained from the New South Wales (NSW) Population and Health Services Research Ethics Committee (HREC/10/CIPHS/33; Cl NSW Study Reference 2010/05/234), the University of NSW Human Research Ethics Committee and the Australian National University Human Research Ethics Committee (2015/513). Participants in the 45 and Up Study provided signed consent for linkage of their information to a range of health-related databases.

Provenance and peer review Not commissioned; externally peer reviewed.

Data sharing statement The datasets used during the current study are available on application to the Sax Institute (www.saxinstitute.org.au/our-work/45-up-study). To do so, one must have a scientifically sound and feasible research proposal, ethics approval for the proposal, data custodian approval for access to linked data and be able to meet the 45 and Up Study license and SURE user charges.

Open access This is an open access article distributed in accordance with the Creative Commons Attribution Non Commercial (CC BY-NC 4.0) license, which permits others to distribute, remix, adapt, build upon this work non-commercially, and license their derivative works on different terms, provided the original work is properly cited, appropriate credit is given, any changes made indicated, and the use is non-commercial. See: http://creativecommons.org/licenses/by-nc/4.0/.

\section{REFERENCES}

1. GBD 2013 Mortality and Causes of Death Collaborators. Global, regional, and national age-sex specific all-cause and causespecific mortality for 240 causes of death, 1990-2013: a systematic analysis for the Global Burden of Disease Study 2013. Lancet 2015;385:117-71.

2. Australian Bureau of Statistics. Causes of Death, Australia, 2016. Cat no. 3303.0. Canberra: ABS, 2017.

3. Australian Institute for Health and Welfare. Cardiovascular disease: Australian facts 2011. Cardiovascular disease series. Cat. no. CVD 53. Canberra: AlHW, 2011.

4. Pagidipati NJ, Peterson ED. Acute coronary syndromes in women and men. Nat Rev Cardiol 2016;13:471-80.

5. Mehta LS, Beckie TM, DeVon HA, et al. Acute myocardial infarction in women: a scientific statement from the American Heart Association. Circulation 2016;133.

6. Maas AH, Appelman YE. Gender differences in coronary heart disease. Neth Heart J 2010;18:598-603.
7. Bogaev RC. Gender Disparities Across the Spectrum of Advanced Cardiac Therapies: Real or Imagined? Curr Cardiol Rep 2016;18:108.

8. Graham G. Acute coronary syndromes in women: recent treatment trends and outcomes. Clin Med Insights Cardiol 2016;10:CMC S37145.

9. Australian Institute of Health and Welfare. Women and heart disease: cardiovascular profile of women in Australia. Cardiovascular disease series no 33 Cat no CVD 49. Canberra: AlHW, 2010.

10. Banks E, Redman S, Jorm L, et al. Cohort profile: the 45 and up study. Int J Epidemiol 2008;37:941-7.

11. The Sax Institute. Questionnaires, 2008.

12. Alexandrescu R, Bottle A, Jarman B, et al. Current ICD10 codes are insufficient to clearly distinguish acute myocardial infarction type: a descriptive study. BMC Health Serv Res 2013;13:468

13. National Centre for Classification in Health. The Australian classification of health interventions (ACHI). 6th edition. Sydney: Tabular list of interventions and alphabetic index of interventions, 2007.

14. Smith DP, Weber MF, Soga K, et al. Relationship between lifestyle and health factors and severe lower urinary tract symptoms (LUTS) in 106,435 middle-aged and older Australian men: population-based study. PLoS One 2014;9:e109278.

15. Kessler RC, Andrews G, Colpe LJ, et al. Short screening scales to monitor population prevalences and trends in non-specific psychological distress. Psychol Med 2002;32:959-76.

16. Quan $\mathrm{H}$, Li B, Couris CM, et al. Updating and validating the Charlson comorbidity index and score for risk adjustment in hospital discharge abstracts using data from 6 countries. Am J Epidemiol 2011;173:676-82.

17. StataCorp. Stata Statistical Software: Release 14. College Station, TX: StataCorp L, 2015

18. Haglund B, Köster M, Nilsson T, et al. Inequality in access to coronary revascularization in Sweden. Scand Cardiovasc J 2004;38:334-9.

19. Raine RA, Black NA, Bowker TJ, et al. Gender differences in the management and outcome of patients with acute coronary artery disease. J Epidemiol Community Health 2002;56:791-7.

20. Redberg RF. Gender differences in acute coronary syndrome: invasive versus conservative approach. Cardiol Rev 2006;14:299-302.

21. Anand SS, Xie CC, Mehta S, et al. Differences in the management and prognosis of women and men who suffer from acute coronary syndromes. J Am Coll Cardiol 2005;46:1845-51.

22. Agewall S, Beltrame JF, Reynolds HR, et al. ESC working group position paper on myocardial infarction with non-obstructive coronary arteries. Eur Heart J 2017;38:143-53.

23. Smilowitz NR, Mahajan AM, Roe MT, et al. Mortality of Myocardial Infarction by Sex, Age, and Obstructive Coronary Artery Disease Status in the ACTION Registry-GWTG (Acute Coronary Treatment and Intervention Outcomes Network Registry-Get With the Guidelines). Circ Cardiovasc Qual Outcomes 2017;10:e003443.

24. Shaw LJ, Shaw RE, Merz CN, et al. Impact of ethnicity and gender differences on angiographic coronary artery disease prevalence and in-hospital mortality in the American College of Cardiology-National Cardiovascular Data Registry. Circulation 2008;117:1787-801.

25. Sheifer SE, Escarce JJ, Schulman KA. Race and sex differences in the management of coronary artery disease. Am Heart $J$ 2000;139:848-57.

26. FitzGerald C, Hurst S. Implicit bias in healthcare professionals: a systematic review. BMC Med Ethics 2017;18:19.

27. Korda RJ, Clements MS, Kelman CW. Universal health care no guarantee of equity: comparison of socioeconomic inequalities in the receipt of coronary procedures in patients with acute myocardial infarction and angina. BMC Public Health 2009;9:460.

28. McCormick N, Lacaille D, Bhole V, et al. Validity of myocardial infarction diagnoses in administrative databases: a systematic review. PLoS One 2014;9:e92286.

29. Heckbert SR, Kooperberg C, Safford MM, et al. Comparison of selfreport, hospital discharge codes, and adjudication of cardiovascular events in the Women's Health Initiative. Am J Epidemiol 2004;160:1152-8.

30. Lindsted KD, Fraser GE, Steinkohl M, et al. Healthy volunteer effect in a cohort study: temporal resolution in the Adventist Health Study. $J$ Clin Epidemiol 1996;49:783-90.

31. Mealing NM, Banks E, Jorm LR, et al. Investigation of relative risk estimates from studies of the same population with contrasting response rates and designs. BMC Med Res Methodol 2010;10:26.

32. Rothman KJ, Gallacher JE, Hatch EE. Why representativeness should be avoided. Int J Epidemiol 2013;42:1012-4. 